\title{
Quantum dot resonant tunneling FET on graphene
}

\author{
Hakimeh Mohammadpour \\ Department of Physics, Azarbaijan Shahid Madani University, 53714-161, Tabriz, Iran
}

\begin{abstract}
At this paper a field effect transistor based on graphene nanoribbon (GNR) is modeled. Like in most GNR-FETs the GNR is chosen to be semiconductor with a gap, through which the current passes at on state of the device. The regions at the two ends of GNR are highly n-type doped and play the role of metallic reservoirs so called source and drain contacts. Two dielectric layers are placed on top and bottom of the GNR and a metallic gate is located on its top above the channel region. At this paper it is assumed that the gate length is less than the channel length so that the two ends of the channel region are un-gated. As a result of this geometry, the two un-gated regions of channel act as quantum barriers between channel and the contacts. By applying gate voltage, discrete energy levels are generated in channel and resonant tunneling transport occurs via these levels. By solving the NEGF and 3D Poisson equations self consistently, we have obtained electron density, potential profile and current. The current variations with the gate voltage give rise to negative transconductance (NTC).
\end{abstract}

Keywords: graphene FET, Quantum Dot, NTC, Resonant tunneling, NEGF

\section{Introduction}

Negative transconductance (NTC) has wide applications in both digital and analog electronics and has been invoked in many quantum electronic devices specially at tunneling transistors and diodes. In consideration of the NTC, nano-electronic devices benefit from the quantum behavior that appears mainly due to device dimensions. Among the many devices aimed at this goal, are the graphene-based structures [1-5]. Ballistic transport of carriers in 
graphene in addition to its two dimensional flat structure makes graphene a promising candidate for observing NTC. In previous researches the NTC has been obtained in several ways. In ref. [1] the resonant tunneling of Dirac fermions through a boron nitride barrier sandwiched between two graphene electrodes is reported. In [6] resonant current peak in a symmetric Graphene p-n layer is obtained which is controlled by chemical doping and applied gate bias. The effect is also revealed at GNR-FET with Oblique top gate [7]. A large peak-to-valley ratio is reported in vertical hetero-structures transistors [8]. Resonant tunneling devices based on the spin-resolved splitting of energy levels in the presence of spin-orbit interaction has also emerged [9-14]. At our previous work we have modeled a pnpGNR-FET in which the pn barriers result in discrete energy levels and resonant tunneling current [15].

In this paper, a new GNR-FET model is proposed in which NTC due to the resonant tunneling is observed. The system is an all-graphene source-channel-drain structure sandwiched between two dielectric layers. The two ends of the GNR are highly doped and behave as reservoirs called source and drain electrodes. Two gates are located at the outer sides of the dielectric layers above the channel region. In this way, two end regions of the graphene ribbon in contact to the reservoirs are not covered by gate electrode. These layers act as barriers and give rise to discrete energy levels at the channel. The calculated current is transmitted by resonant tunneling through these levels and exhibit NTC.

\section{Device Model and Simulation Approach}

At this section the model device structure and physics of its function is explained. It is composed of an armchair graphene nanoribbon (A-GNR) that is sandwiched between two dielectric layers. The layers are assumed to be $\mathrm{SiO}_{2}$ with dielectric constant of about $k=3.9$. In this kind of FET systems the source and drain contacts as well as the channel are formed on a single graphene layer (fig. 1). Therefore an ohmic contact between the channel and each of the reservoirs is formed and the transistor acts as traditional MOSFETs.

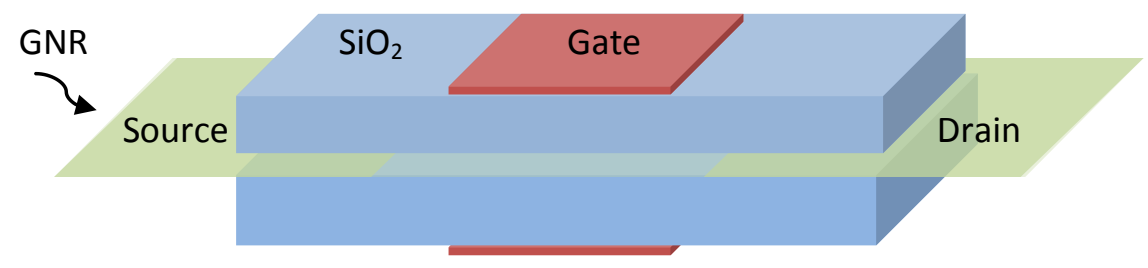


Fig. 1: Schematic representation of the MOSFET-like GNR-FET.

In order to get on/off states as the main characteristics of transistor, the ribbon is required to be gap-full at the central or channel region. It is known that GNRs of zigzag-type edges don't provide energy gap and thus are practically unsuitable for usage as channel of transistor. On the other hand armchair GNRs gather semiconducting band gap in some widths of the ribbon. A-GNRs with number of unit cells in the width direction equal to $N_{W}=3 \mathrm{~m}$ or $3 m+1$ ( $m$ being an integer number) reveal energy gap that increases with decreasing ribbon width (by decreasing $m$ ).

At this model, as applied in many previous papers, the conducting source and drain regions are obtained by highly doping the ends of the GNR [16-18].

The gate metals are separated from the ribbon above the central region by two dielectric layers. This geometry which is provided by the $2 \mathrm{D}$ structure of graphene and its good contact to the $\mathrm{SiO}_{2}$ layer has been used by many researches and has resonant or traditional MOSFET current-voltage characteristics depending on the parameters of system.

The distinct feature of this paper that we added to the above mentioned structure is a new design of gates that give rise to resonant tunneling through the device. Here, the gate electrodes of GNR-FET (fig. 1) cover partially the quasi-one dimensional channel at the center and leaves the two ends intact. That means the two regions of graphene channel which are connected to the source and drain are un-gated semiconductors. Hence, when the gate voltage is applied, the two ends of the channel are not affected and in this way don't provide any energy level around the Fermi energy. These ungated gap-full regions act as barriers between the reservoirs and channel region that may under suitable conditions give rise to the discrete energy levels at channel. This is the phenomenon responsible for resonant tunneling through the channel. The isolated channel at our model, gathers the quantum dot behavior by discrete energy levels. 
Each of the GNR reservoirs consists of 30 unit cells and the channel length, indexed by the number of one dimensional unit cells of channel, $N_{C h}$ (as indicated at ref. [15]) takes different amounts. In order to obtain suitable band gap, the AGNR of width $N_{W}=9$ is chosen.

The charge in and current through the channel are computed by the non-equilibrium Green's function (NEGF) formalism with nearest neighbor tight-binding Hamiltonian with an atomistic $\mathrm{p}_{\mathrm{z}}$-orbital basis-set, self-consistently solved by the 3-dimensional Poisson equation [19].

In the real space NEGF formalism the retarded Green's function of the device is defined as;

$$
G(E)=\left[(E+i \eta) I-H-U-\sum_{s}-\sum_{d}\right]^{-1}
$$

Where $E$ is energy and $U$ is a diagonal matrix, whose diagonal entries are the on-site potential energy and $H=-t \sum_{i, j} c_{i}^{\dagger} c_{j}$ is the tight-binding Hamiltonian of the simulated region where $i, j$ stand for nearest neighbors as well as $c_{i}^{\dagger}$ and $c_{j}$ stand for creation and annihilation operators at sites $i$ and $\mathrm{j}$ respectively. The effects of rest of the semi-infinite source and drain are included through the self-energies, $\Sigma_{s}$ and $\Sigma_{d}$, which are calculated according to the iterative algorithm described in [20]. $\eta=10^{-5}(\mathrm{eV})$ is assumed and $t=2.7 \mathrm{eV}$ is the hopping energy.

$U$ is a diagonal matrix, with diagonal entries representing on-site potential energies. This matrix is obtained by self-consistent computing the charge density and the electrode potentials from the Poisson and NEGF equations. The electron (n) density is calculated from the following relations;

$$
n=2 e \int_{0}^{+\infty} \frac{d E}{2 \pi} G^{n}(E)
$$

Where $G^{n}(E)=G \sum^{i n} G^{\dagger}$ is called electron correlation function. $\sum^{\text {in }}$ represents the inscattering and out-scattering functions of the contacts and is determined by;

$$
\sum^{\text {in }}(E)=\Gamma_{\mathrm{s}}(E) \mathrm{f}_{\mathrm{s}}(E)+\Gamma_{\mathrm{d}}(E) \mathrm{f}_{\mathrm{d}}(E)
$$

$\Gamma_{\mathrm{s}(\mathrm{d})}=\mathrm{i}\left(\sum_{\mathrm{s}(\mathrm{d})}-\sum_{\mathrm{s}(\mathrm{d})}^{\dagger}\right)$ represents channel level broadening due to the source (s) and drain (d) contacts and $f_{s(d)}(E)$ are Fermi energies at contacts.

The retarded Green's function $G(E)$ is calculated from relation (1) in which, the potential matrix, $U$, is required. To this end Poisson equation in the form $\vec{\nabla} \cdot\left(K \varepsilon_{0} \overrightarrow{\nabla U}\right)=n(\vec{r}) e^{2}$ should be solved which demands knowledge of the function $n(\vec{r})$ in the channel. In this 
equation $\varepsilon_{0}$ is the permittivity of vacuum and the dielectric constant $K$ is 3.9 and $e$ is the electron charge that in electron-volt unit equals to 1 . So, a self-consistent procedure is required for obtaining $n$ and $U$. Starting from an initial guess for the potential energy profile, the Green's equation is solved and the resulting electron density is fed into Poisson equation for obtaining new $U$. This cycle is repeated until the self-consistency for $U$ and $n$ is achieved.

After the self-consistency is achieved and the desired Green's function is obtained the current within the device is calculated by;

$$
I=\frac{2 e}{\hbar} \int_{-\infty}^{+\infty} d E \bar{T}(E)\left(f_{s}(E)-f_{d}(E)\right), \quad \bar{T}(E)=\operatorname{trace}\left[\Gamma_{\mathrm{s}} G \Gamma_{\mathrm{d}} G^{\dagger}\right]
$$

where $\bar{T}(E)$ is the transmission function.

Discussion: The promised resonant tunneling current (at small bias voltages) stem from discreteness of the energy levels at channel. This is due to the confinement restriction on the channel by the lateral barriers in contact to the reservoirs. Different parameters of the device contribute to the channel characteristics. It is evident that the larger energy level separation results in the best performance of the resonant tunneling devices such as RTT, resonant tunneling diodes (RTD) and the high frequency applications. To this end the ribbon energy gap $\left(E_{\mathrm{g}}\right)$, which is determined by the ribbon width $\left(\mathrm{N}_{\mathrm{W}}\right)$, separating barriers widths $\left(L_{\text {Barrier }}\right)$, doping concentration of the reservoirs in molar units (doping), the gate length ( $\left.L_{G a t e},\right)$ and the channel length $\left(L_{C h}\right)$ which contains the whole un-doped regions between two reservoirs (such that $L_{C h}=L_{\text {Gate }}+L_{\text {Barrier }}$ ) are involved. At the next section, the results are declared.

\section{Results and discussion}


Let's first recall the parameters that are not introduced yet. $L_{S, D}$ are the source and drain lengths that are incorporated in the Poisson equation and are assigned equal sizes at this paper. $L_{\text {Diel. }}$ stands for width of each of dielectric layers in the direction normal to the graphene layer. $V_{G}$ and $V_{D}$ are the gate and the drain voltages, respectively, measured with respect to the pre-assumed grounded source. We note that all the length characteristics of the device along the ribbon are presented in terms of the number of unit cells as well as in all the diagrams we take $N_{S(D)}=30$ (in terms of the number of unit cells) by which the flat band condition at the two far ends of reservoirs are guaranteed. Unit cell of an AGNR as depicted at [15] contains a rectangle that contains a Benzene ring at the length direction and extended in the width direction to contain all the rings at that direction, so that the ribbon may be assumed a one dimensional chain of this rectangular unit cells.

The first system that is considered has the following parameters: $L_{C h}=40, L_{\text {Barrier }}=5$, $L_{\text {Diel. }}=2 \mathrm{~nm}$, doping $=0.02, N_{W}=9, E_{g} \approx 0.96 \mathrm{eV}$.

The edges of the conduction and valence bands are plot after self-consistent procedure for these parameters at Fig. 2.

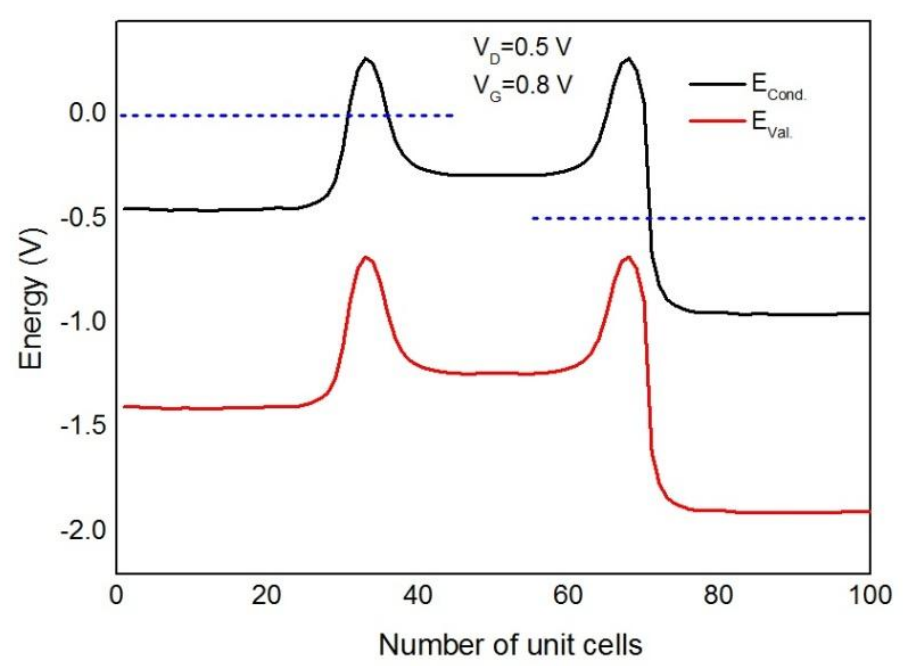

Fig. 2: The conduction (black) and valence (red) band profiles along the channel for the assigned voltages. The semi-Fermi levels are shown in dashed lines. 
To illustrate the gate effect on the depth of constructed quantum dot at the channel between the barriers, potential profile is plot for two $V_{G}$ s in figure 3 .

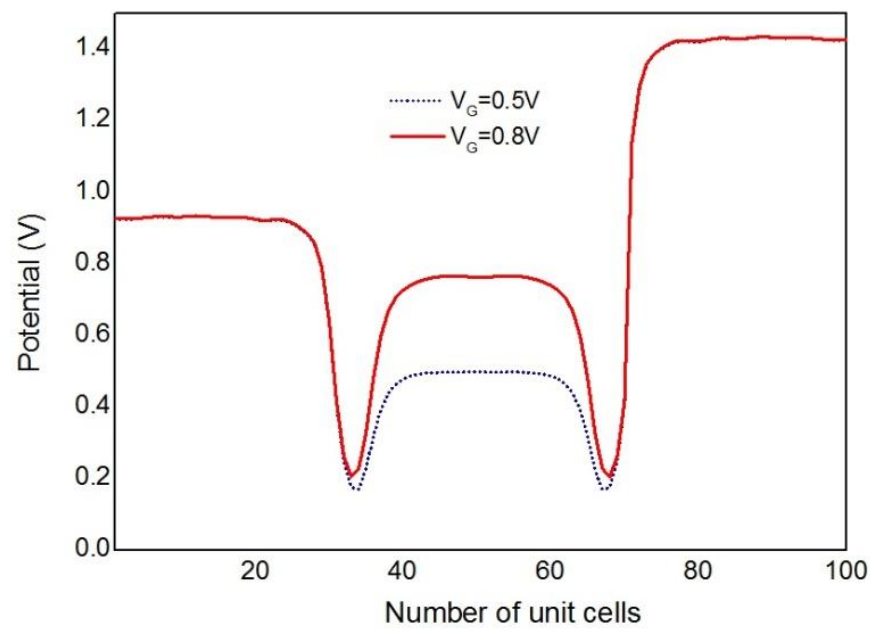

Fig. 3: Potential profile for $V_{\mathrm{G}}=0.5 \mathrm{~V}, 0.8 \mathrm{~V}$. The depth of quantum well increases by $V_{\mathrm{G}}$.

The $I-V_{D}$ graphs for different amounts of $V_{\mathrm{G}}$ are depicted in figure 4 .

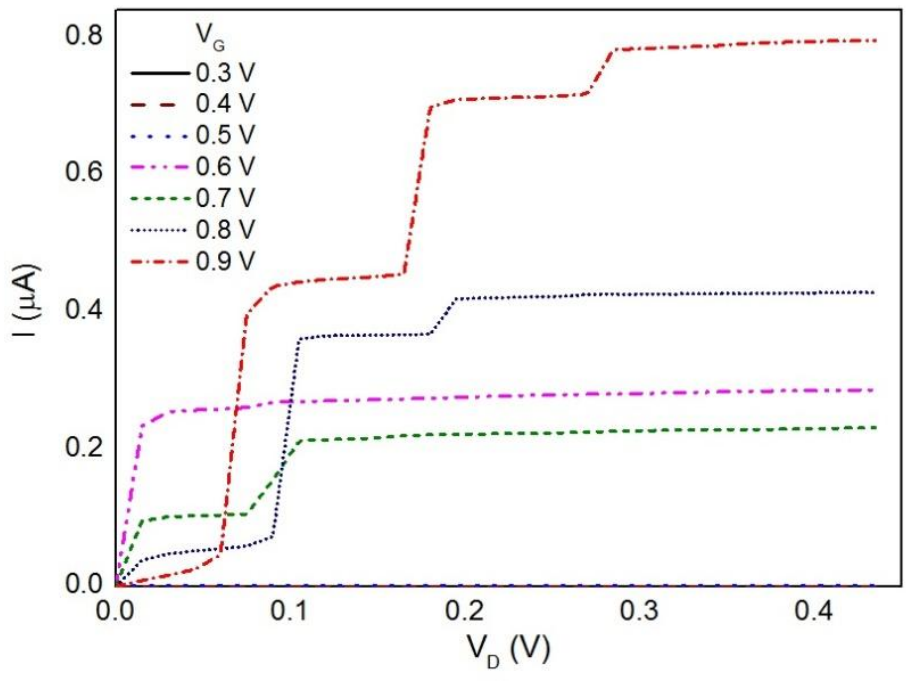

Fig. 4: The $I-V_{D}$ curves for various $V_{\mathrm{G}} \mathrm{s}$. The rise of pronounced current for $V_{\mathrm{G}} \geq 0.6(\mathrm{~V})$ and step-like increase of current are observed. 
The calculated step-like $I-V_{D}$ diagram is due to the discreteness of the channel energy levels which is the characteristic behavior of resonant tunneling devices.

By investigating the diagrams, the non-uniform behavior of the current by for different gate voltages $V_{\mathrm{G}}$ in a typical small $V_{D}$ is pronounced. To track this behavior, we have calculated $I-V_{G}$ for some values of $V_{D}$ and plot the results at figure 5 .

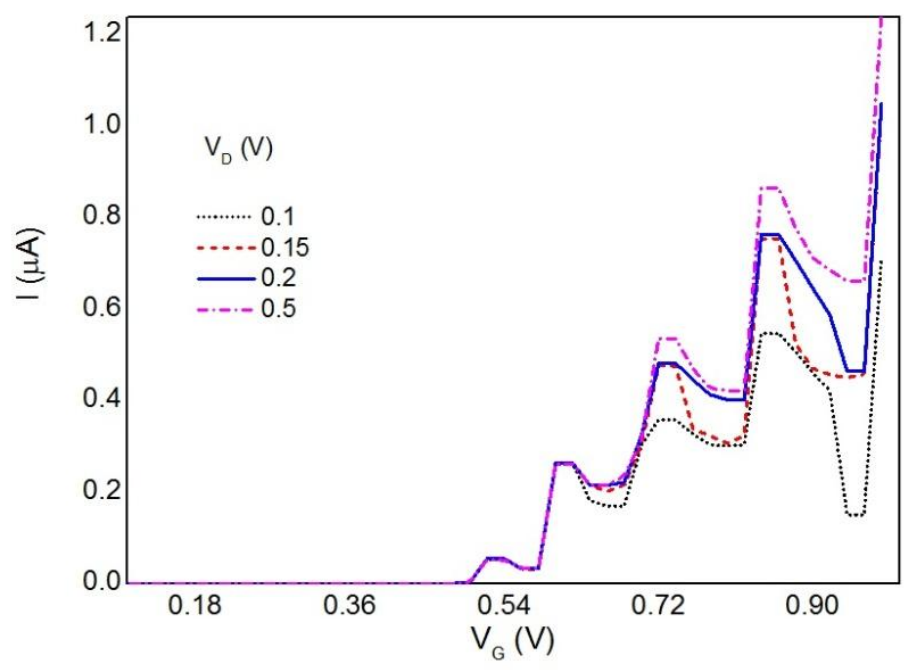

Fig. 5: $I-V_{G}$ for typical amounts of $V_{D}$. The larger PVR at smaller $V_{D}$ are observed.

Besides the overall increase of the current the oscillations with the gate voltage is the expected behavior of this research. Hence, the negative transconductance at some voltages with peak to valley ratio (PVR) of about 3 are obtained.

The strength of the level discreteness and so PVR, are determined by the isolation quality of the channel quantum dot. In this way the barriers' length as well as the channel length for a determined width play major roles.

The above calculations are repeated for $L_{C h}=50$ and the corresponding diagrams are plot in figures 6 and 7. 


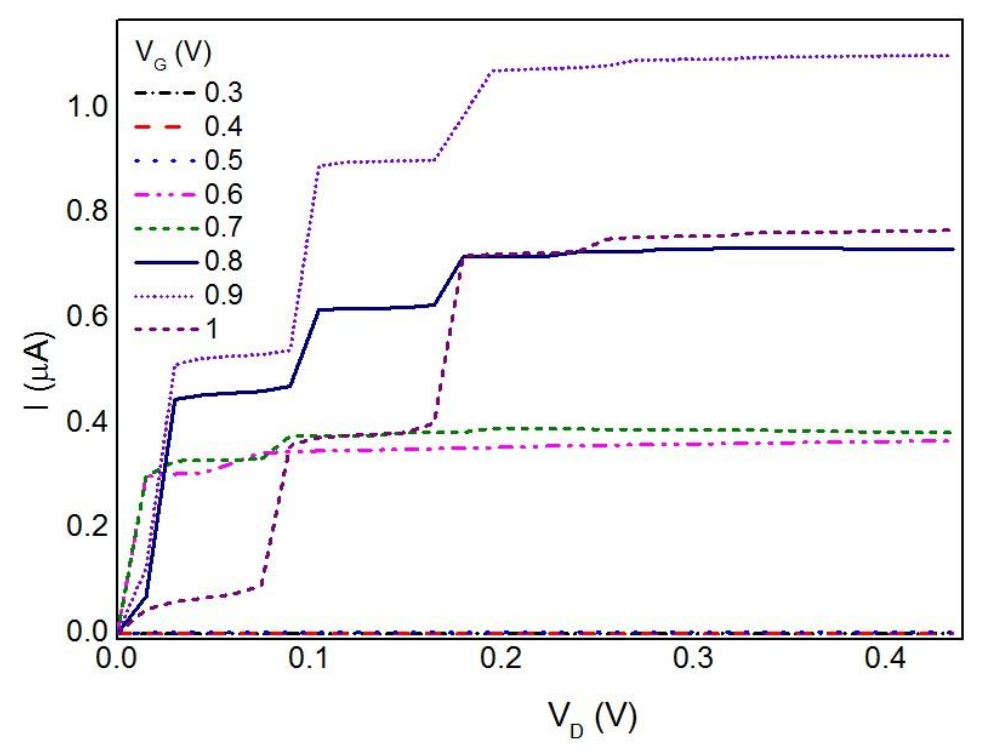

Fig. 6: $I-V_{D}$ curves for $L_{C h}=50$ and various $V_{\mathrm{G}} \mathrm{s}$.

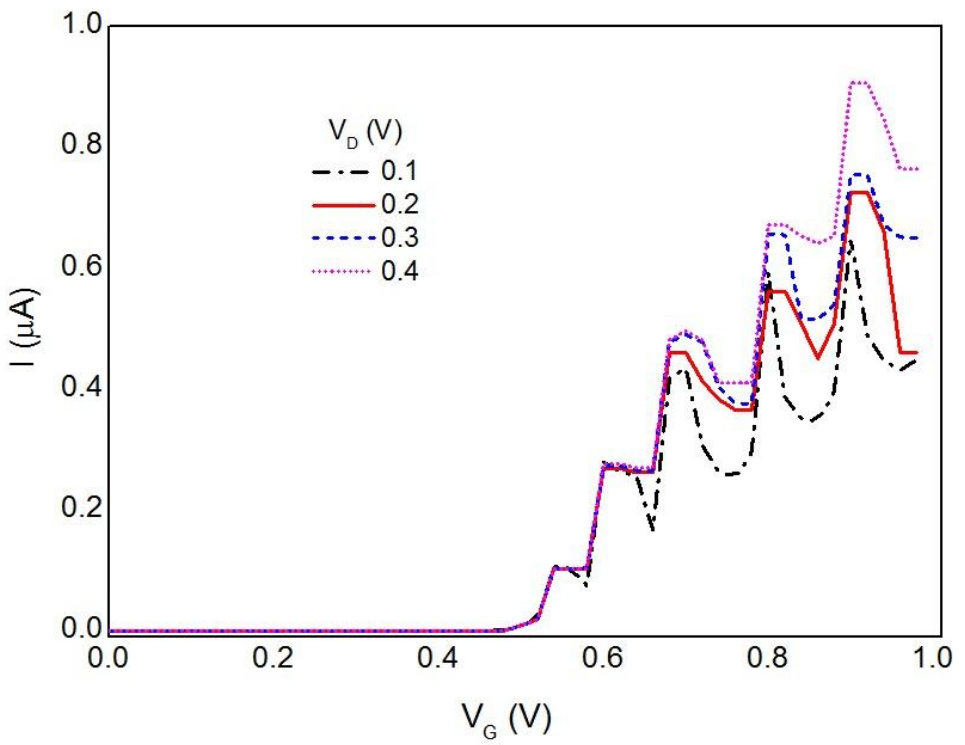

Fig. 7: $I-V_{G}$ curves for $L_{C h}=50$ and various $V_{\mathrm{D}} \mathrm{s}$.

To investigate the effect of $L_{\text {Barrier }}$, the resultant current calculated for $L_{\text {Barrier }}=7$ and $L_{C h}=40$ is presented at the following section.

The conduction and valence bands profiles along the channel are plot at fig. 8 . 


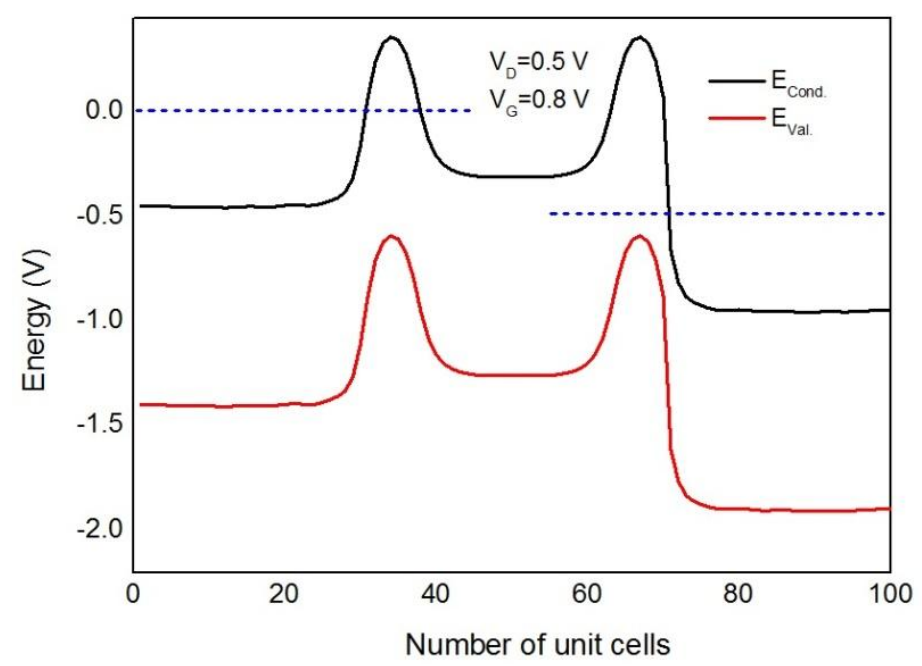

Fig. 8: The conduction and valence bands along channel for assigned voltages and the same parameters of fig. 2 for $L_{\text {Barrier }}=7, L_{C h}=40$.

The $I-V_{D}$ curves for different amounts of $V_{\mathrm{G}}$ are plot at figure 8 .

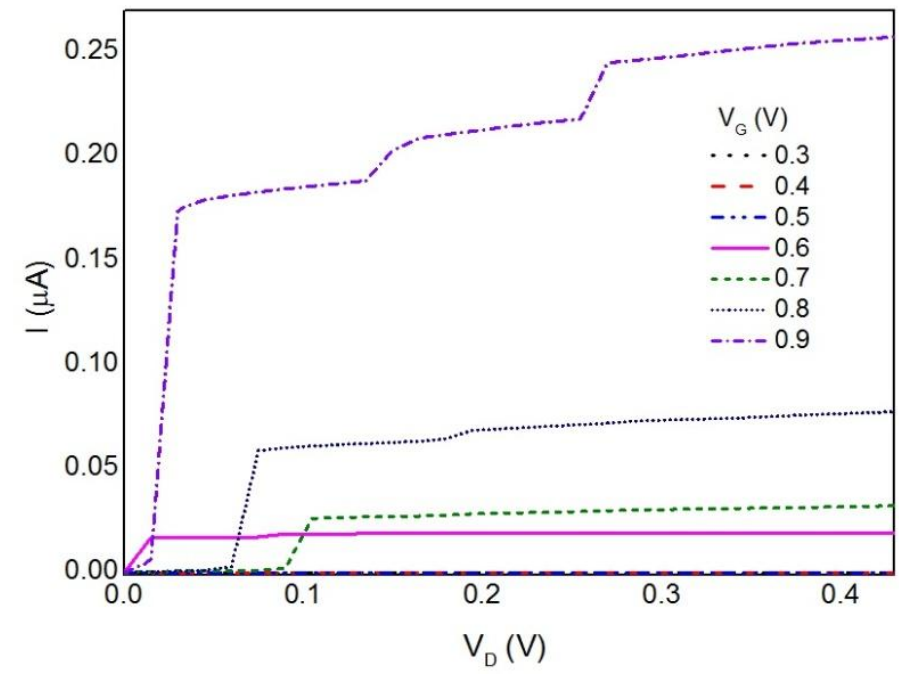

Fig. 9: The $I-V_{D}$ curves for various $V_{\mathrm{G}} \mathrm{s}$ for $L_{C h}=40, L_{\text {Barrier }}=7$.

The $I-V_{G}$ for some values of $V_{D}$ are plot at figure 9 . 


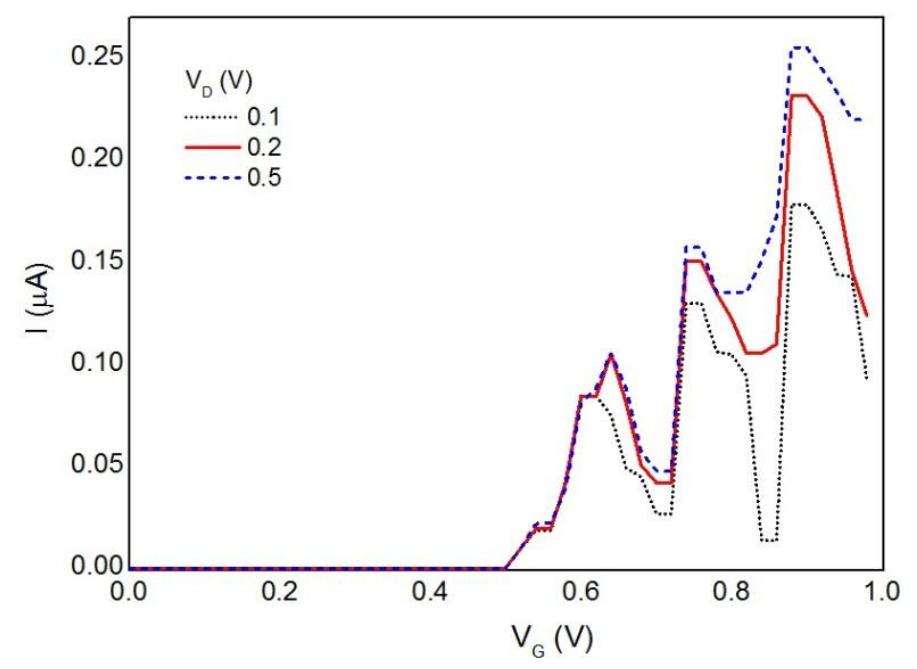

Fig. 10: The $I-V_{G}$ curves for various $V_{\mathrm{D}} \mathrm{s}$ for $L_{C h}=40, L_{\text {Barrier }}=7$.

At this point we show the discrete channel levels by considering local density of states. In figure 11 the density of states color plot is presented along channel as a function of energy for $V_{G}=0.8(V)$ and $V_{D}=0$.



Fig. 11: The density of states color plot along channel for different energies.

For completeness let's show the transmission function, T(E), color plot versus the gate voltage and energy. In figure 12 the horizontal axis is gate voltage and the vertical axis is 
energy. The transmission function at discrete energies for gate voltages above some threshold is observed which more clarifies the source of NTC. The minimum of the energy at which transmission occurs is shifted towards low energies by increasing the gate voltage which is a manifestation for increasing the depth of the quantum well by the gate voltage.

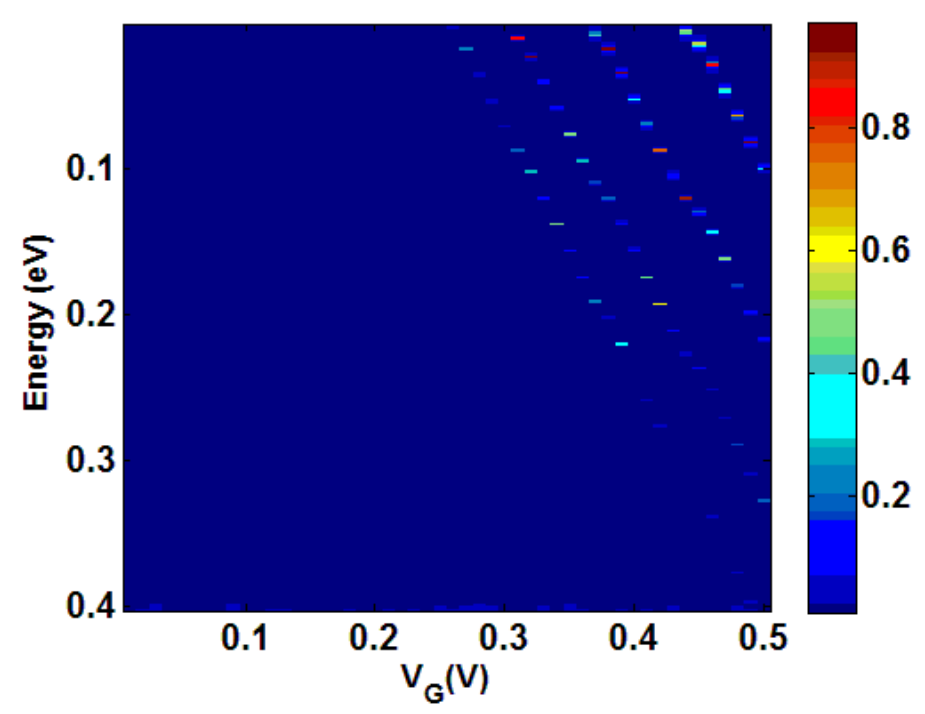

Fig. 12: $\mathrm{T}(\mathrm{E})$ image versus the gate voltage and energy for $L_{C h}=40, L_{\text {Barrier }}=7$.

\section{Conclusion}

A new design for GNR-FET is modeled in which against to the traditional GNR-FETs the gate metals cover just the middle part of the channel layer. In this way two barriers are constructed at the contact between the reservoirs (source and drain) and the channel. This confinement of the semiconductor channel by the barriers forms a quantum dot channel and hence gives rise to discreteness of energy levels. The calculated current that is established by resonant tunneling through the discrete energy levels manifests NTC.

\section{Acknowledgment}

This research is supported by Iran National Science Foundation (INSF), Number 9203633. 


\section{References}

[1] L. Britnell, R. V. Gorbachev, A. K. Geim, et al., NATURE COMMUNICATIONS, 4, Article number: 1794, 2013.

[2] G. Fiori, IEEE Electron Device Letters, Vol. 32, p. 1334--1336, 2011.

[3] V. N. Do, A. V. H. Nguyen, P. Dollfus, et al., J. Appl. Phys. ,vol.104,no.6,pp.063708-1063708-7, Sep. 2008.

[4] . Dragoman and M. Dragoman, Appl Phys Lett.,vol.90,no.14,pp.143111-1-143111-3, Apr. 2007.

[5] R. Nandkishore and L. Levitov, Proc. Natl. Acad. Sci. 108, 14021-14025 (2011). [6] Pei Zhao, R. M. Feenstraz, G. Gu, et al., arXiv:1301.0673.

[7] M. Dragoman, A. Dinescu, D. Dragoman, arXiv:1405.6407.

[8] P. M. Campbell, A. Tarasov, C. A. Joiner,et al., ACS Nano, 2015, 9 (5), pp 5000-5008.

[9] Y.A. Bychkov, E.I. Rashba, C Solid State 17 (1984) 6039.

[10] Goran Isić, et al., J. Appl. Phys. 108 (2010) 044506.

[11] David Z.-Y. Ting, et al., Proc. IEEE 91 (2003) 5.

[12] Wu Qing-Ping, et al., Appl. Phys. Lett. 105 (2014) 252402.

[13] A. Iovan, et al., Nano Lett. 8 (3) (2008) 805-809.

[14] H. Mohammadpour, Journal of Magnetism and Magnetic Materials 385 (2015) 129-132.

[15] H. Mohammadpour and A. Asgari, Physica E 46 (2012) 270-273.

[16] H. Mohammadpour, A. Asgari, Physica E-Low-Dimensional Systems and Nanostructures (2011) 43.

[17] R. Grassi, S. Poli, E. Gnani, et al., Solid-State Electronics 53 (2009) 462.

[18] R. Grassi, A. Gnudi, S. Reggiani, et al., Journal of Computational Electronics 8 (2009) 441.

[19] K.I. Bolotin, K.J. Sikes, Z. Zhang, et al., Solid State Communications 146 (9/10) (2008) 351.

[20] M.P. Lopez Sancho, J.M. Lopez Sancho, J. Rubio, Journal of Physics F (1985) 15. 